\title{
Sensing Metabolic Signals with Nascent RNA Transcripts: The T Box and S Box Riboswitches as Paradigms
}

\author{
T.M. HENKIN AND F.J. GRUNDY \\ Department of Microbiology and The RNA Group, The Ohio State University, Columbus, Ohio 43210
}

\begin{abstract}
Recent studies in a variety of bacterial systems have revealed a number of regulatory systems in which the $5^{\prime}$ region of a gene plays a key role in regulation of the downstream coding sequences. These RNA regions act in cis to determine if the full-length transcript will be synthesized or if the coding sequence(s) will be translated. Each class of system includes an RNA element whose structure is modulated in response to a specific regulatory signal, and the signals measured can include small molecules, small RNAs (including tRNA), and physical parameters such as temperature. Multiple sets of genes can be regulated by a particular mechanism, and multiple systems of this type, each of which responds to a specific signal, can be present in a single organism. In addition, different classes of RNA elements can be found that respond to a particular signal, indicating the existence of multiple alternate solutions to the same regulatory problem. The T box and $\mathrm{S}$ box systems, which respond to uncharged tRNA and $S$-adenosylmethionine (SAM), respectively, provide paradigms of two systems of this type.
\end{abstract}

Direct sensing of a regulatory signal by the $5^{\prime}$ region of a nascent RNA (the "leader region") has recently emerged as a common mechanism for regulation of gene expression in bacteria (Grundy and Henkin 2004, 2006). In mechanisms of this type, termed "riboswitches," the regulatory signal can modulate folding of the nascent RNA transcript, which in turn can determine whether the RNA folds into the helix of an intrinsic transcriptional terminator, resulting in premature termination of transcription, or an alternate structure that allows expression of the downstream coding sequences (Fig. 1). Similar RNA rearrangements can also mediate translational regulation by sequestration of the ribosome-binding site at the start of the regulated coding sequence. Each class of riboswitch RNA recognizes its regulatory signal with high specificity, and the RNA structural elements exhibit a sensitivity to the signal that is appropriate to the in vivo pools of the effector or to the physical parameter (e.g., temperature). In addition, the RNA structural change that occurs in response to the signal must be coupled to an appropri-

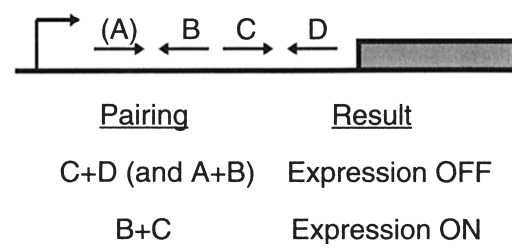

Figure 1. Regulation of gene expression by leader RNA structural rearrangements. Pairing of the RNA regions labeled $\mathrm{C}$ and $D$ results in inhibition of downstream gene expression by formation of the helix of an intrinsic transcriptional terminator or a structure that sequesters the ribosome-binding site of the coding sequence. Formation of the competing $\mathrm{B}+\mathrm{C}$ pairing sequesters region $\mathrm{C}$, preventing transcription termination or translation inhibition. The $\mathrm{A}+\mathrm{B}$ pairing, which occurs in some systems (e.g., $\mathrm{S}$ box) but not others (e.g., T box) competes with the $\mathrm{B}+\mathrm{C}$ pairing, thereby controlling formation of the $\mathrm{C}+\mathrm{D}$ pairing. ately sensitive gene expression response. We have identified several systems of this type, including the T box system, which monitors the aminoacylation of a specific tRNA, and the $\mathrm{S}$ box and $\mathrm{S}_{\mathrm{MK}}$ box systems, which respond directly to SAM.

Characterization of the RNA-effector interaction in these systems has provided new information about how different classes of effectors are recognized, and about the impact of these regulatory mechanisms on the cell.

\section{THE T BOX SYSTEM: REGULATION OF AMINO ACID-RELATED GENES BY UNCHARGED TRNA}

The $\mathrm{T}$ box system was initially uncovered by the analysis of the Bacillus subtilis tyrS gene, which encodes tyrosyl-tRNA synthetase. A G+C-rich helix followed by a run of $\mathrm{U}$ residues was identified upstream of the $t y r S$-coding sequence, leading to the prediction that regulation occurs at the level of premature termination of transcription. Expression of tyrS was shown to be induced when cells were grown with limiting tyrosine, and regulation was dependent on the presence of the terminator, as predicted (Henkin et al. 1992). The 5' region of the tyrS gene shares a set of sequence and structural elements with a large family of aminoacyl-tRNA synthetase, amino acid biosynthesis, and transporter genes (Grundy and Henkin 1993, 1994, 2003). These features form a pattern that includes the transcriptional terminator and a competing antiterminator structure, preceded by a large stem-loop element interrupted by several bulges and internal loops (Fig. 2). One of these loops contains a single codon that corresponds to the amino acid identity of the downstream gene, so that the $t y r S$ gene contains a UAC tyrosine codon, tryptophanyl genes contain a UGG tryptophan codon, etc. (Grundy and Henkin 1993). The initial pattern was recognized from a set of ten aminoacyl-tRNA synthetase genes from Bacillus sp., and the explosion of genome sequence 

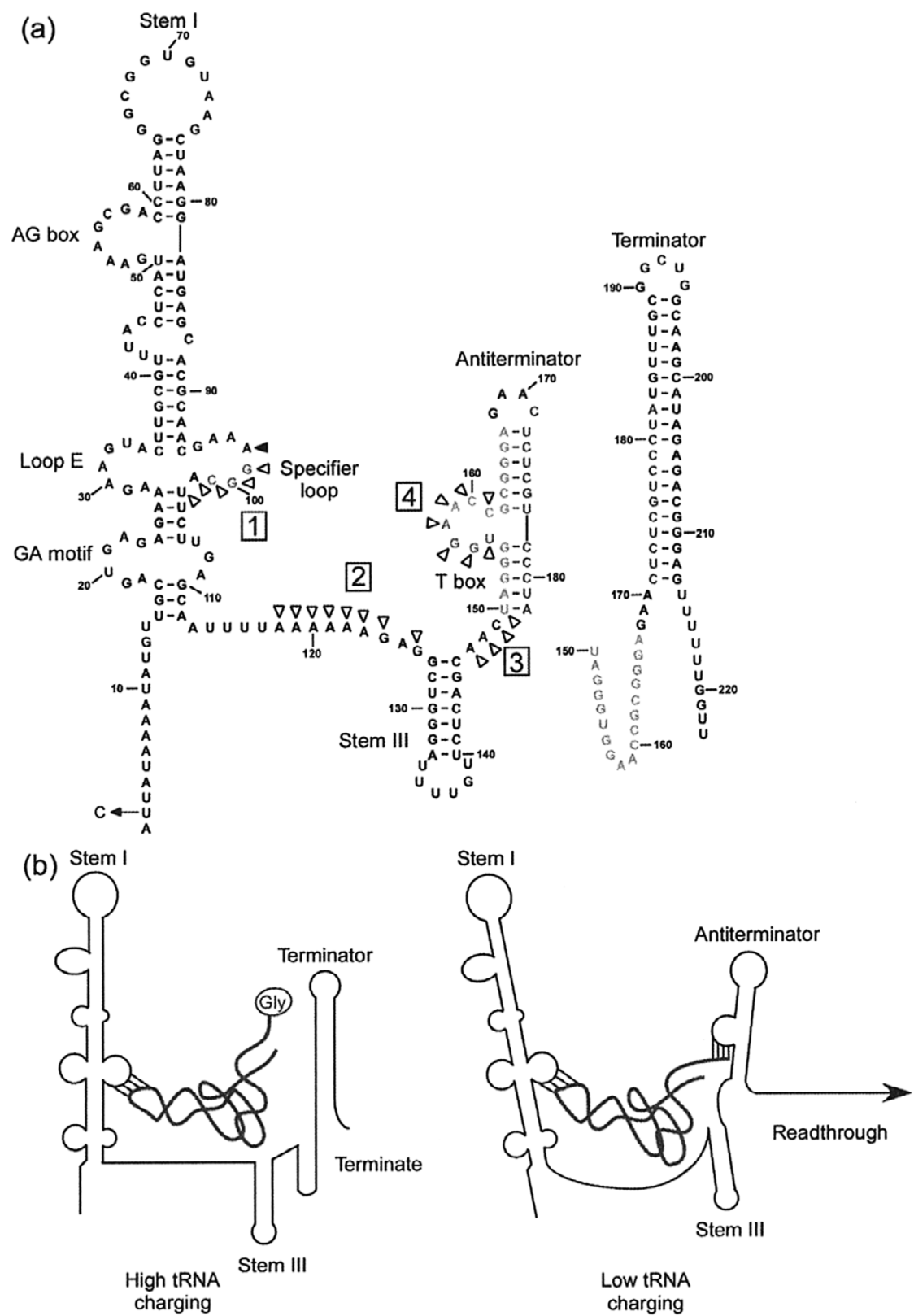

Figure 2. The T box system. (a) Secondary structural model of the B. subtilis glyQS leader RNA in the antiterminator conformation; the competing terminator conformation is shown on the right. Numbering is relative to the transcription start site. Major structural elements are labeled. Boxed numbers and arrows indicate regions that exhibit alterations in structure upon binding of the cognate tRNA $^{\text {Gly }}$ (Yousef et al. 2005). The GGC residues in the specifier loop (in addition to the following A residue) pair with the anticodon loop of tRNA ${ }^{\text {Gly }}$. (b) Both charged and uncharged tRNA interact with the specifier loop of the leader RNA; only uncharged tRNA can make the second interaction between the acceptor end of the tRNA and the antiterminator bulge. The second interaction stabilizes the antiterminator, preventing formation of the competing terminator helix. Conditions under which the effector tRNA is highly charged result in efficient termination and low expression of the downstream coding sequence, whereas a decrease in charging of the effector tRNA results in readthrough of the termination site and increased expression of the downstream coding sequence. Each gene responds specifically to the cognate tRNA.

information has expanded the set to more than 700 genes, all of which exhibit correspondence between the identity of the codon at the appropriate position and the predicted function of the downstream coding sequence (Merino and Yanofsky 2005; F.J. Grundy and T.M. Henkin, unpubl.). The identification of this RNA pattern suggested that amino acid limitation could be sensed via an effect on charging of the cognate tRNA.

\section{Genetic Analysis of the B. subtilis tyrS Gene}

The prediction that the codon was responsible for the specificity of the response to amino acid limitation was tested by mutation of the UAC tyrosine codon of the tyrS gene to a UUC phenylalanine codon. This singlenucleotide substitution resulted in loss of response to tyrosine availability and induction of tyrS expression when 
cells were limited for phenylalanine. Replacement of the UAC tyrosine codon with a nonsense codon resulted in transcription termination under all growth conditions, and expression was restored by introduction into the cell of a nonsense suppressor tRNA containing a compensatory anticodon mutation (Grundy and Henkin 1993). These studies established that base-pairing between the sequence at the position of the UAC codon in tyrS (designated the "specifier sequence") and the anticodon of the corresponding tRNA is required for readthrough of the terminator in the $5^{\prime}$ region of the gene and suggested that each gene containing the $\mathrm{T}$ box leader region pattern would respond similarly to its cognate tRNA. This prediction was subsequently tested for a variety of genes in this family.

An obvious mechanism for utilization of a codon within the $5^{\prime}$ region of an mRNA as a regulatory signal is provided by transcription attenuation systems similar to the Escherichia coli trp operon, where regulatory codons are monitored by a translating ribosome (Landick et al. 1996). In systems of this type, which rely on coupling of transcription and translation, the regulated genes are preceded by a short peptide-coding sequence that includes tandem codons for the corresponding amino acid (e.g., tryptophan codons for the trp biosynthesis operon). Efficient translation of the leader peptide-coding sequence requires an adequate supply of the appropriate charged tRNA (e.g., tRNA ${ }^{T r p}$ ), and stalling of the ribosome because of limitation for specific charged tRNAs results in formation of an antiterminator structure in the nascent RNA that prevents formation of the helix of an intrinsic transcriptional terminator, therefore preventing premature termination of transcription. This type of mechanism was ruled out for the $B$. subtilis tyrS gene when introduction of a frameshift mutation, which was predicted to disrupt translation of the tyrosine codon, had no effect on $t y r S$ regulation, indicating that the UAC codon does not act through translation.

Induction of tyr $S$ expression by limitation for tyrosine suggested that antitermination occurs preferentially under conditions when aminoacylation of $\mathrm{RNA}^{\mathrm{Tyr}}$ is reduced, whereas termination occurs when tRNA ${ }^{\mathrm{Tyr}}$ is efficiently aminoacylated. Since tyr $S$ encodes the enzyme responsible for aminoacylation of $\mathrm{tRNA}^{\mathrm{Tyr}}$, this regulatory pattern would allow increased synthesis of the enzyme in response to an increase in the substrate:product ratio. Expression of an unchargeable variant of tRNA ${ }^{\mathrm{Tyr}}$ in vivo resulted in efficient readthrough of the tyr $S$ leader region terminator during growth in the presence of tyrosine, demonstrating that uncharged tRNA is the effector in vivo and that amino acid limitation acts through its effect on tRNA aminoacylation (Grundy et al. 1994). Nonsense suppressor tRNAs were further used to demonstrate that the presence in the cell of charged tRNA matching the specifier sequence reduces the efficiency of antitermination directed by uncharged tRNA, supporting the model that the system responds to the ratio between charged and uncharged tRNA, rather than the absolute amount of uncharged tRNA.

The molecular basis for discrimination between uncharged and charged tRNA was also established by genetic analyses. Possible base-pairing between the unpaired acceptor end of uncharged tRNA ( $5^{\prime}$-NCCA-3') and residues within the bulge of the antiterminator ele- ment (5'-UGGN-3') was supported by covariation between the variable position of the antiterminator (the $\mathrm{N}$ position in the bulge) and the tRNA discriminator position (preceding the 3 '-terminal CCA). Substitution of the variable position in the $t y r S$ antiterminator bulge resulted in loss of expression, despite the fact that this position is not conserved in other genes in the $\mathrm{T}$ box family. Restoration of antitermination by a compensatory change in tRNA $^{\mathrm{Tyr}}$ supported the model that base-pairing is required, and suggested that addition of an amino acid to the $3^{\prime}$ end of the tRNA could interfere with its interaction with the antiterminator (Grundy et al. 1994).

Extensive mutational analysis of both the tyrS leader sequence and $\mathrm{tRNA}^{\mathrm{Tyr}}$ demonstrated that the sequence and structural elements conserved in T box leader RNAs are important for antitermination in vivo (Rollins et al. 1997; Winkler et al. 2001; Grundy et al. 2002b) and that the overall tertiary structure of the tRNA is required, although substitutions within helical domains and in the variable loop of the tRNA are tolerated (Grundy et al. 2000). Modification of the tyrS specifier sequence and antiterminator to permit interaction with noncognate tRNAs revealed that although certain tRNAs could be directed to interact with the tyrS leader RNA in vivo, other tRNAs exhibit no antitermination activity, and no tRNA is as effective as the cognate tRNA (Grundy et al. 1997). These results suggested the existence of recognition determinants in addition to the specifier sequence and the variable position of the antiterminator, but no clear pattern emerged from bioinformatics analysis of $\mathrm{T}$ box leaders and the corresponding tRNAs (F.J. Grundy and T.M. Henkin, unpubl.).

\section{Biochemical Analysis of the T Box System}

Although genetic analyses of the B. subtilis tyrS gene, and similar analyses of other $\mathrm{T}$ box genes, clearly demonstrated that interaction of the leader RNA with the cognate uncharged tRNA is required for antitermination, a key unanswered question was whether the tRNA-leader RNA interaction is sufficient to promote antitermination in the absence of other cellular factors. A small model RNA designed to mimic the antiterminator element was capable of specific tRNA acceptor end binding (Gerdeman et al. 2002), whereas an RNA modeled on the specifier loop region of the B. subtilis glyQS leader sequence could bind an anticodon stem-loop RNA (Nelson et al. 2006). However, tRNA interaction with an intact tyrS leader could not be demonstrated (Grundy et al. 2002b; F.J. Grundy and T.M. Henkin, unpubl.).

The B. subtilis glyQS leader RNA, a natural variant that lacks two large structural elements common to most $\mathrm{T}$ box leaders, was employed in an attempt to reproduce tRNAdependent antitermination in vitro. A simplified transcription system utilizing purified components, including either B. subtilis or E. coli RNAP, was used to demonstrate that the glyQS leader region terminator is active in vitro; addition of tRNA ${ }^{\text {Gly }}$ generated by in vitro transcription with phage T7 RNAP is sufficient to promote efficient antitermination in the absence of other cellular components (Grundy et al. 2002a). Antitermination in vitro requires base-pairing between the tRNA anticodon and acceptor 
end with the glyQS specifier sequence and antiterminator bulge, as was observed in vivo. These studies clearly demonstrated that the nascent RNA is competent for specific tRNA binding and can discriminate between cognate and noncognate tRNAs. Mutational analysis of tRNA Gly showed that the intact tRNA is required (Yousef et al. 2003), as was previously observed in vivo. $\mathrm{RNA}^{\mathrm{Thr}}$-dependent antitermination of the more complex B. subtilis thrS gene in vitro was demonstrated by Putzer et al. (2002), but additional components were required. Charged tRNA (for $t h r S$ ) or addition of an extra nucleotide at the $3^{\prime}$ end of the tRNA (for gly $Q S$ ) inhibits antitermination, as predicted by the in vivo studies, and the charged tRNA (or charged tRNA mimic) acts as a competitive inhibitor (Putzer et al. 2002; Grundy et al. 2005). Transcription complexes were probed with an antisense DNA oligonucleotide complementary to sequences that form the $3^{\prime}$ side of the terminator helix, and binding of the oligonucleotide was monitored by cleavage with RNase $\mathrm{H}$, which is specific for RNADNA hybrids (Yousef et al. 2005). These studies clearly demonstrated that complexes generated in the absence of tRNA or in the presence of the charged tRNA mimic are in the terminator configuration (resistant to oligonucleotide binding and RNase $\mathrm{H}$ cleavage), whereas complexes generated in the presence of uncharged tRNA are in the antiterminator configuration (sensitive to RNase $\mathrm{H}$ ).

Since two positions of base-pairing between the tRNA and the leader RNA were required for antitermination, a set of transcription complexes in which transcription elongation could be transiently blocked at specific positions by binding of a DNA-binding protein to the template DNA was generated to assess the ability of uncharged tRNA ${ }^{\text {Gly }}$ and a charged tRNA ${ }^{\text {Gly }}$ mimic to interact with the nascent transcript. Early during transcription, either tRNA could interact with the transcript, and the efficiency of antitermination was dependent on the ratio of the two tRNA species; preincubation with uncharged tRNA yielded complexes that could be disrupted by addition of the charged tRNA mimic (Grundy et al. 2005). Complexes containing the entire leader RNA, including the intact antiterminator, were fully competent for interaction with uncharged tRNA, but these complexes were now resistant to challenge with the charged tRNA mimic. These results suggested that pairing between the acceptor end of the tRNA and the antiterminator results in formation of a stable complex that can no longer be disrupted by an excess of charged tRNA.

The ability of a nascent transcript extending through the antiterminator to interact with tRNA suggested the possibility that $g l y Q S$ leader RNA synthesized in vitro by T7 RNAP could also be competent for tRNA binding. This was tested using a size exclusion filtration assay, which permitted separation of tRNA-leader RNA complexes from unbound tRNA. Binding was dependent on basepairing with the specifier sequence and the antiterminator and required denaturation of the leader RNA and refolding in the presence of the tRNA (Yousef et al. 2005).

\section{T Box Leader RNA Structure}

Formation of leader RNA-tRNA complexes in vitro was exploited to map changes in cleavage patterns using a variety of structure-sensitive RNA cleavage agents. The
glyQS leader RNA exhibited cleavage patterns consistent with the predicted structure, although several regions of the RNA shown as unpaired loops in the phylogenetic model were resistant to cleavage, suggesting that these regions are in fact structured (Yousef et al. 2005); these results are consistent with predictions that several unpaired domains within $\mathrm{T}$ box family leaders form higher-order structural domains, including kink-turn and $\mathrm{S}$-turn motifs. Incubation in the presence of tRNA Gly results in protection of the specifier sequence region and the antiterminator bulge, consistent with the known leader RNA-tRNA base-pairing in those regions. Protection of the anticodon loop of the tRNA, and of D19 in the D loop, was also observed. Additional regions of the leader RNA that are not known to directly interact with the tRNA were also protected in the presence of the matching uncharged tRNA, whereas no protection was observed with a mismatched tRNA, and only the specifier region was protected by the charged tRNA mimic (Fig. 1) (Yousef et al. 2005; N. Green et al., unpubl.). These results are consistent with genetic data which suggest that regions of the leader in addition to the specifier sequence and antiterminator are important for antitermination in vivo. The observation that charged tRNA confers protection only of the specifier sequence supports the model that although both uncharged and charged tRNA can make the initial interaction with the specifier sequence, only uncharged tRNA can interact with the antiterminator, and that interaction is required for stabilization of the tRNA-leader RNA complex and antitermination.

Detailed structural information is currently available only for the antiterminator domain of T box leader RNAs. The nuclear magnetic resonance (NMR) structure of a 39nucleotide antiterminator model RNA was determined (Gerdeman et al. 2003), revealing stacking of the upper helix and the 3 ' portion of the internal bulge onto the bottom helix. The bulge residues predicted to interact with the acceptor end of the tRNA appear to be highly flexible, suggesting that these residues are made available for interaction with the tRNA, allowing stabilization of the antiterminator element by tRNA binding. Alteration of a conserved $\mathrm{C}$ residue in the 3 ' portion of the bulge resulted in an increase in mobility of the bulge region and reduction of tRNA binding to the model RNA, consistent with the deleterious effect of the corresponding substitution on antitermination in vivo and in vitro in the context of an intact leader RNA (Rollins et al. 1997; N. Green et al., unpubl.; J. Hines, pers. comm.). These results provide further support for the importance of the arrangement of the bulge residues for antiterminator function.

A key open question is the arrangement of the Stem I region that includes the specifier sequence, which is crucial for specific recognition of the anticodon loop of the cognate tRNA. Genetic studies have demonstrated the importance of conserved elements within Stem I, including the kink-turn motif below the specifier loop, the Sturn within the specifier loop, and the AG loop and GGUGNRA elements at the top (Rollins et al. 1997; Winkler et al. 2001; N. Green et al., unpubl.). A combination of genetic, biochemical, and structural biology approaches will be necessary to provide insight into the role of Stem I elements in tRNA binding. 


\section{Transcriptional versus Translational Control}

Most $\mathrm{T}$ box family genes are found in low $\mathrm{G}+\mathrm{C}$ grampositive bacteria, but there are also a number of genes that have been identified in high $\mathrm{G}+\mathrm{C}$ gram-positives as well as certain gram-negative organisms (Grundy and Henkin 2003; Merino and Yanofsky 2005). In these cases, regulation appears to operate at the level of translation initiation, rather than transcription termination, since the final helical element of the RNA does not resemble an intrinsic transcriptional terminator but instead is positioned to sequester the Shine-Dalgarno (SD) sequence, so that formation of this helix is predicted to inhibit binding of the 30S ribosomal subunit to the mRNA. T box RNAs of this type are predicted to form an alternate structure that is stabilized by binding to the cognate tRNA; in this case, the alternate structure sequesters sequences that would otherwise bind to the SD region, so that binding of uncharged tRNA allows translation of the downstream coding sequence. No systems of this type have yet been characterized biochemically, so that translational control remains to be demonstrated experimentally.

\section{THE S BOX SYSTEM: REGULATION OF METHIONINE-RELATED GENES BY SAM}

Initial analysis of the distribution of $\mathrm{T}$ box genes in bacterial genomes revealed that although genes involved in methionine biosynthesis were members of the T box family in certain organisms, such as Enterococcus, no $\mathrm{T}$ box genes with AUG methionine codons were uncovered in the first genomes of Bacillus and Clostridium that were available (although genes of this type were subsequently uncovered; Grundy and Henkin 2002; Rodionov et al. 2004). Multiple genes involved in biosynthesis and transport of methionine were instead found to contain leader RNA elements that fit a new pattern designated the $\mathrm{S}$ box (Fig. 3) (Grundy and Henkin 1998). The identification of this pattern upstream of the SAM synthetase gene in several organisms suggested that SAM, which is synthesized from methionine, was the likely final product of the regulated pathway. The role of SAM as the effector was supported by the observation that $B$. subtilis mutants with defects in SAM synthetase exhibited elevated methionine pools, whereas overexpression of SAM synthetase resulted in methionine auxotrophy (Wabiko et al. 1988; Yocum et al. 1996). Transcriptional analysis of B. subtilis gene expression during growth with various sulfur sources also led to the prediction that SAM is the likely effector for regulation of methionine biosynthesis (Auger et al. 2002). A combination of genetic and biochemical studies, recently capped by the three-dimensional structure of an S box RNA in complex with SAM (Montange and Batey 2006), has now established the molecular mechanism for SAM-dependent repression of S box gene expression.

\section{Genetic Analysis of S Box RNAs}

Like T box RNAs, most $\mathrm{S}$ box sequences include transcriptional terminators and competing antiterminators. However, in this case, the terminator helices were predicted to be less stable than the antiterminator elements,

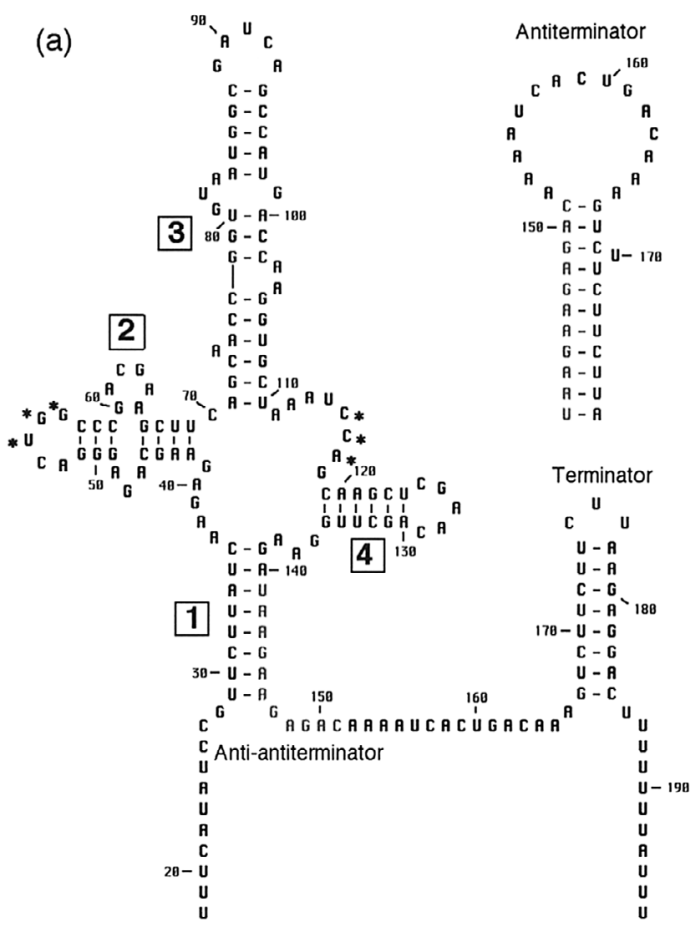

(b)

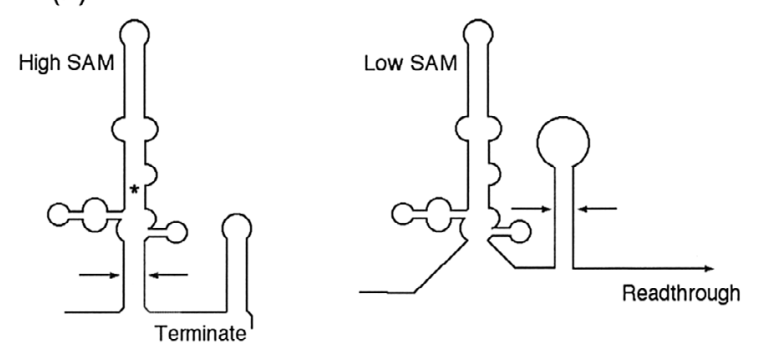

Figure 3. The S box system. (a) Secondary structural model of the $B$. subtilis yitJ leader RNA in the terminator conformation; the competing antiterminator conformation is shown above the terminator. The anti-antiterminator, which forms in conjunction with the terminator, sequesters sequences (gray) that form the $5^{\prime}$ side of the antiterminator. Numbering is relative to the transcription start site. Boxed numbers indicate helices $1-4$ of the SAM-binding element. Asterisks indicate residues that pair to form a tertiary interaction that is facilitated by the kink-turn in the internal bulge of helix 2. (b) Helix 1 is stabilized by binding of SAM (asterisk within helix 3; the position of SAM has been demonstrated by crystal structure analysis of the SAM-RNA complex [Montange and Batey 2006]). Formation of helix 1 prevents formation of the antiterminator, allowing formation of the competing terminator helix and premature termination of transcription. In the absence of SAM, the RNA folds into the more stable antiterminator conformation, and transcription proceeds past the termination site, allowing expression of the downstream coding regions.

and the sequences forming the $5^{\prime}$ side of the antiterminator were predicted to pair with sequences further upstream, to form an anti-antiterminator element, formation of which would prevent formation of the antiterminator. These predictions were tested by mutational analysis using the B. subtilis yitJ gene, which encodes methylenetetrahydrofolate reductase.

Expression of yitJ was shown to be low when cells were grown in the presence of high methionine and was 
induced when cells were starved for methionine; this response was dependent on the presence of the terminator, and mutations that disrupted the antiterminator resulted in low, uninducible expression. In contrast, mutations that disrupted the anti-antiterminator resulted in high expression during growth in the presence of methionine, consistent with the prediction that this element competes with formation of the antiterminator to cause repression (Grundy and Henkin 1998). The anti-antiterminator element forms the base of a complex four-way helical junction that includes a pseudoknot, the arrangement of which is highly conserved in S box RNAs. Disruption of any of the conserved sequence or structural elements within the $\mathrm{S}$ box pattern (with the exception of the antiterminator) resulted in high expression during growth in methionine, indicating that these elements are required for repression (Grundy and Henkin 1998; Winkler et al. 2001; McDaniel et al. 2005). B. subtilis strains with elevated or reduced SAM synthetase activity in vivo exhibited alterations in $\mathrm{S}$ box gene expression, consistent with the model that SAM is the effector in vivo (McDaniel et al. 2003, 2006).

\section{Biochemical Analyses of S Box RNAs}

A direct interaction between SAM and S box RNAs was reported by three groups (Epshtein et al. 2003; McDaniel et al. 2003; Winkler et al. 2003). The S box leader RNA terminator element was shown to be inactive in an in vitro transcription system using purified components, and addition of SAM was sufficient to cause efficient termination in the absence of other cellular components. SAM binding resulted in rearrangement of the RNA from the antiterminator to the anti-antiterminator conformation, in agreement with the genetic studies which suggested that the anti-antiterminator element is the target for binding of the regulatory factor that promotes termination in vivo. Binding of SAM to the purified RNA was also demonstrated, and mutations that disrupt repression in vivo resulted in loss of SAM binding and loss of the SAM-dependent transcription termination in vitro. SAM binds with very high affinity to purified $\mathrm{S}$ box leader RNAs, and different $\mathrm{S}$ box genes exhibit different sensitivities to SAM both in vitro and in vivo, in a pattern consistent with the physiological role of the regulated gene products (J. Tomsic et al., unpubl.). The SAM-RNA complex is also very stable in vitro and is unlikely to dissociate within the time frame of the termination/antitermination decision.

\section{Structural Analysis of S Box RNAs}

A variety of techniques have been employed to monitor changes in S box RNA structure in response to SAM. Structural mapping using oligonucleotide binding (Epshtein et al. 2003; McDaniel et al. 2003), in-line attack (Winkler et al. 2003), and enzymatic cleavage (McDaniel et al. 2005) all showed stabilization of the anti-antiterminator element by SAM binding and multiple changes throughout the S box RNA, including stabilization of the tertiary interaction between the loop of helix 2 and the helix 2-3 junction region (McDaniel et al. 2005). These results were recently substantiated by the crystal structure of an S box RNA in complex with SAM, which revealed a compact structure with SAM embedded in a pocket formed by the juxtaposition of helices 1 and 3 and stabilized by the tertiary interaction (Montange and Batey 2006). Like other riboswitch RNAs, the SAM-binding S box RNA exploits the entire surface of the effector molecule to mediate specific recognition.

\section{Transcriptional versus Translational Control}

As has been found for a number of other riboswitch RNAs, regulation by the $\mathrm{S}$ box element appears to occur predominantly at the level of premature termination of transcription in low $\mathrm{G}+\mathrm{C}$ gram-positive bacteria, but it can also occur at the level of translation initiation (Grundy and Henkin 2006). In genes of this type, the final helix that is predicted to form in the presence of SAM includes the SD sequence of the downstream coding sequence, whereas in the absence of SAM, a competing helix (analogous to the antiterminator described above) prevents formation of the SD-sequestering helix. Translational control is unusual among $\mathrm{S}$ box genes and appears to predominate in high $\mathrm{G}+\mathrm{C}$ gram-positive and in gram-negative species.

\section{Other SAM-binding Riboswitch RNAs}

Two classes of SAM-binding RNAs distinct from the S box RNAs have been uncovered. The SAM-II RNAs are found in $\alpha$-proteobacteria, but their mechanism of action is unknown (Corbino et al. 2005). The $\mathrm{S}_{\mathrm{MK}}$ box riboswitch is found in SAM synthetase (metK) genes of lactic acid bacteria including Streptococcus and Enterococcus sp., and SAM binding was shown to cause sequestration of the SD region in a pseudoknot structure that represses translation initiation in vivo (Fuchs et al. 2006). It therefore appears that there are multiple molecular mechanisms for SAM recognition by RNA and that regulation of genes involved in generation of methionine and SAM can respond to SAM pools in different ways.

\section{CONCLUSION}

The abundance of systems in which nascent RNAs act in cis to modulate expression of downstream coding sequences is growing rapidly. Although regulation at the levels of premature termination of transcription and translation initiation predominate so far, it is likely that more examples of other mechanisms, including effects on RNA stability, will be discovered. For some of these mechanisms, including translational regulation, it is possible that the regulatory elements can act in full-length rather than nascent transcripts, and that the elements can be located in regions other than the $5^{\prime}$ end of the mRNA. Uncovering novel variations on this general theme will require both new bioinformatics approaches and the ability to recognize alternative possibilities that deviate from the paradigm systems. Biophysical approaches are begin- 
ning to complement the genetics and biochemistry that have revealed so much of the basic mechanisms underlying these systems, and the emerging three-dimensional structures provide new insight into the molecular recognition properties of regulatory RNAs. The possibility that many more mechanisms of this type may operate in eukaryotes is also tantalizing.

\section{ACKNOWLEDGMENTS}

This work was supported by National Institutes of Health grants GM47823 and GM63615.

\section{REFERENCES}

Auger S., Danchin A., and Martin-Verstraete I. 2002. Global expression profile of Bacillus subtilis grown in the presence of sulfate or methionine. J. Bacteriol. 81: 741.

Corbino K.A., Barrick J.E., Lim J., Welz R., Tucker B.J., Puskarz I., Mandal M., Rudnick N.D., and Breaker R.R. 2005. Evidence for a second class of $S$-adenosylmethionine riboswitches and other regulatory RNA motifs in alpha-proteobacteria. Genome Biol. 6: R70.

Epshtein V., Mironov A.S., and Nudler E. 2003. The riboswitchmediated control of sulfur metabolism in bacteria. Proc. Natl. Acad. Sci. 100: 5052.

Fuchs R.T., Grundy F.J., and Henkin T.M. 2006. The $\mathrm{S}_{\mathrm{MK}}$ box is a new SAM-binding RNA for translational regulation of SAM synthetase. Nat. Struct. Mol. Biol. 13: 226.

Gerdeman M.S., Henkin T.M., and Hines J.V. 2002. In vitro structure-function studies of the Bacillus subtilis tyrS mRNA antiterminator: Evidence for factor-independent tRNA acceptor stem binding specificity. Nucleic Acids Res. 30: 1065.

. 2003. Solution structure of the Bacillus subtilis T-box antiterminator RNA: Seven nucleotide bulge characterized by stacking and flexibility. J. Mol. Biol. 326: 189.

Grundy F.J. and Henkin T.M. 1993. tRNA as a positive regulator of transcription antitermination in B. subtilis. Cell 74: 475 .

- 1994. Conservation of a transcription antitermination mechanism in aminoacyl-tRNA synthetase and amino acid biosynthesis genes in Gram-positive bacteria. J. Mol. Biol. 235: 798 .

- 1998. The S box regulon: A new global transcription termination control system for methionine and cysteine biosynthesis genes in Gram-positive bacteria. Mol. Microbiol. 30: 737 .

2002. Synthesis of serine, glycine, cysteine and methionine. In Bacillus subtilis and its closest relatives: From genes to cells (ed. A.L. Sonenshein et al.), p. 245. American Society for Microbiology Press, Washington, D.C.

2003. The T box and $\mathrm{S}$ box transcription termination control systems. Front. Biosci. 8: d20.

2004. Regulation of gene expression by effectors that bind to RNA. Curr. Opin. Microbiol. 7: 126.

. 2006. From ribosome to riboswitch: Control of gene expression in bacteria by RNA structural rearrangements. Crit. Rev. Biochem. Mol. Biol. (in press).

Grundy F.J., Rollins S.M., and Henkin T.M. 1994. Interaction between the acceptor end of tRNA and the T box stimulates antitermination in the Bacillus subtilis tyrS gene: A new role for the discriminator base. J. Bacteriol. 176: 4518.

Grundy F.J., Winkler W.C., and Henkin T.M. 2002a. tRNAmediated transcription antitermination in vitro: Codon-anticodon pairing independent of the ribosome. Proc. Natl. Acad. Sci. 99: 11121.

Grundy F.J., Yousef M.R., and Henkin T.M. 2005. Monitoring uncharged tRNA during transcription of the Bacillus subtilis gly QS gene. J. Mol. Biol. 346: 73.

Grundy F.J., Collins J.A., Rollins S.M., and Henkin T.M. 2000.
tRNA determinants for transcription antitermination of the Bacillus subtilis tyrS gene. RNA 6: 1131 .

Grundy F.J., Hodil S.E., Rollins S.M., and Henkin T.M. 1997. Specificity of tRNA-mRNA interactions in Bacillus subtilis tyrS antitermination. J. Bacteriol. 179: 2587.

Grundy F.J., Moir T.R., Haldeman M.T., and Henkin T.M. $2002 \mathrm{~b}$. Sequence requirements for terminators and antiterminators in the $\mathrm{T}$ box transcription antitermination system: Disparity between conservation and functional requirements. Nucleic Acids Res. 30: 1646.

Henkin T.M., Glass B.L., and Grundy F.J. 1992. Analysis of the Bacillus subtilis tyrS gene: Conservation of a regulatory sequence in multiple tRNA synthetase genes. J. Bacteriol. 174: 1299 .

Landick R., Turnbough C.L., and Yanofsky C. 1996. Transcription attenuation. In Escherichia coli and Salmonella: Cellular and molecular biology (ed. F.C. Neidhardt et al.), p. 1263. American Society for Microbiology, Washington, D.C.

McDaniel B.A.M., Grundy F.J., and Henkin T.M. 2005. A tertiary structural element in S box leader RNAs is required for $S$-adenosylmethionine-directed transcription termination. Mol. Microbiol. 57: 1008.

McDaniel B.A.M., Grundy F.J., Artsimovitch I., and Henkin T.M. 2003. Transcription termination control of the $\mathrm{S}$ box system: Direct measurement of $S$-adenosylmethionine by the leader RNA. Proc. Natl. Acad. Sci. 100: 3083.

McDaniel B.A., Grundy F.J., Kurlekar V.P., Tomsic J., and Henkin T.M. 2006. Identification of a mutation in the Bacillus subtilis $S$-adenosylmethionine synthetase gene that results in derepression of S-box gene expression. J. Bacteriol. 188: 3674 .

Merino E. and Yanofsky C. 2005. Transcription attenuation: A highly conserved regulatory strategy used by bacteria. Trends Genet. 21: 260.

Montange R.K and Batey R.T. 2006. Structure of the $S$-adenosylmethionine riboswitch regulatory mRNA element. Nature 441: 1172 .

Nelson A.R., Henkin T.M., and Agris P.F. 2006. tRNA regulation of gene expression: Interactions of an mRNA $5^{\prime}$-UTR with a regulatory tRNA. RNA 12: 1254 .

Putzer H., Condon C., Brechemier-Baey D., Brito R., and Grunberg-Manago M. 2002. Transfer RNA-mediated antitermination in vitro. Nucleic Acids Res. 30: 3026.

Rodionov D.A., Vitreschak A.G., Mironov A.A., and Gelfand M.S. 2004. Comparative genomics of the methionine metabolism in Gram-positive bacteria: A variety of regulatory systems. Nucleic Acids Res. 32: 3340.

Rollins S.M., Grundy F.J., and Henkin T.M. 1997. Analysis of cis-acting sequence and structural elements required for antitermination of the Bacillus subtilis tyrS gene. Mol. Microbiol. 25: 411.

Wabiko H., Ochi K., Nguyen D.M., Allen E.R., and Freese E. 1988. Genetic mapping and physiological consequences of metE mutations of Bacillus subtilis. J. Bacteriol. 170: 2705.

Winkler W.C., Grundy F.J., Murphy B.A., and Henkin T.M. 2001. The GA motif: An RNA element common to bacterial antitermnation systems, rRNA, and eukaryotic RNAs. RNA 7: 1165.

Winkler W.C., Nahvi A., Surarsan N., Barrick J.E., and Breaker R.R. 2003. An mRNA structure that controls gene expression by binding $S$-adenosylmethionine. Nat. Struct. Biol. 10: 701.

Yocum R.R., Perkins J.B., Howitt C.L., and Pero J. 1996. Cloning and characterization of the metE gene encoding $S$ adenosylmethionine synthetase from Bacillus subtilis. J. Bacteriol. 178: 4604.

Yousef M.R., Grundy F.J., and Henkin T.M. 2003. tRNA requirements for gly $Q S$ antitermination: A new twist on tRNA. RNA 9: 1148 .

. 2005. Structural transitions induced by the interaction between tRNA ${ }^{\text {Gly }}$ and the Bacillus subtilis glyQS T box leader RNA. J. Mol. Biol. 349: 273. 


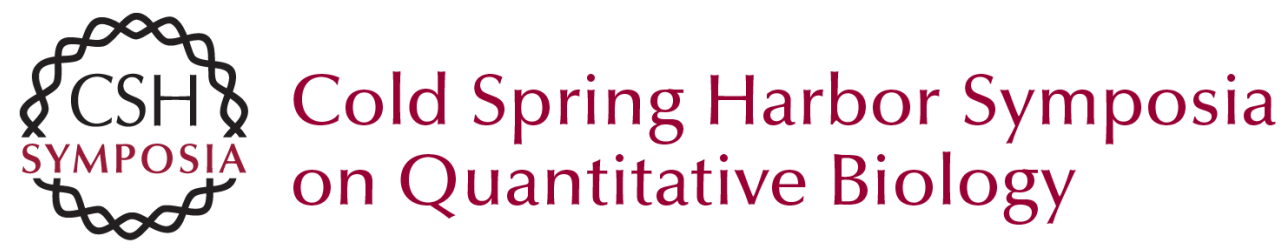

\section{Sensing Metabolic Signals with Nascent RNA Transcripts: The T Box and S Box Riboswitches as Paradigms}

T.M. HENKIN and F.J. GRUNDY

Cold Spring Harb Symp Quant Biol 2006 71: 231-237

Access the most recent version at doi:10.1101/sqb.2006.71.020

References This article cites 33 articles, 13 of which can be accessed free at: http://symposium.cshlp.org/content/71/231.full.html\#ref-list-1

License

Email Alerting Receive free email alerts when new articles cite this article - sign up in Service the box at the top right corner of the article or click here. 